\title{
Green synthesis and characterization of gelatin-based and sugar-reduced silver nanoparticles
}

This article was published in the following Dove Press journal:

International Journal of Nanomedicine

16 March 2011

Number of times this article has been viewed

\author{
Majid Darroudi',2 \\ Mansor Bin Ahmad ${ }^{3}$ \\ Abdul Halim Abdullah ${ }^{1,3}$ \\ Nor Azowa Ibrahim ${ }^{3}$ \\ 'Advanced Materials and \\ Nanotechnology Laboratory, Institute \\ of Advanced Technology (ITMA), \\ Universiti Putra Malaysia, Selangor, \\ Malaysia; ${ }^{2}$ Department of Chemistry, \\ Faculty of Science, Ferdowsi \\ University of Mashhad, Mashhad, Iran; \\ ${ }^{3}$ Department of Chemistry, Faculty \\ of Science, Universiti Putra Malaysia, \\ Selangor, Malaysia
}

Correspondence: Majid Darroudi

Advanced Materials and

Nanotechnology Laboratory,

Institute of Advanced Technology

(ITMA), Universiti Putra Malaysia,

43400 UPM Serdang,

Selangor, Malaysia

Tel +60389466044

Fax +60389466043

Email majiddarroudi@gmail.com

\begin{abstract}
Silver nanoparticles (Ag-NPs) have been successfully prepared with simple and "green" synthesis method by reducing $\mathrm{Ag}^{+}$ions in aqueous gelatin media with and in the absence of glucose as a reducing agent. In this study, gelatin was used for the first time as a reducing and stabilizing agent. The effect of temperature on particle size of Ag-NPs was also studied. It was found that with increasing temperature the size of nanoparticles is decreased. It was found that the particle size of Ag-NPs obtained in gelatin solutions is smaller than in gelatin-glucose solutions, which can be related to the rate of reduction reaction. X-ray diffraction, ultravioletvisible spectra, transmission electron microscopy, and atomic force microscopy revealed the formation of monodispersed Ag-NPs with a narrow particle size distribution.
\end{abstract}

Keywords: silver nanoparticles, green method, gelatin, glucose, UV-vis spectra

\section{Introduction}

Synthesis of nanomaterials with the desired quality and properties is one of the key issues in current nanotechnology. ${ }^{1}$ Today, the "green" synthesis of metallic nanoparticles has received increasing attention due to the development of eco-friendly technologies in materials science. Well-dispersed and ultrafine metal nanoparticles, especially transition metals, have great interest due to their distinctive physicochemical and thermodynamic properties, which have made them suitable for use in various fields, such as catalysis, ${ }^{2}$ optics, ${ }^{3}$ and medicine.$^{4}$ In green synthesis of nanoparticles, three important rules of green chemistry should be considered: (i) choice of the green solvents used in the synthesis, ii) choice of an eco-friendly benign reducing agent, and (iii) choice of a nontoxic material as a stabilizer. To date, most of the preparation methods published are based on using organic materials due to the hydrophobicity of the stabilizing agents used, such as natural polymers. Polymers are a category of macromolecules. Using a polymer as a matrix for preparing metallic nanoparticles, the repeat unit of the polymer should include metal species or have functional groups presented in order to bind the metal. When using polymers as soft support, the diameter of metallic nanoparticles can be logically controlled.

Gelatin is the protein from collagen and has a three-chain helical structure in which individual helical chains are stranded in a super-helix about the common molecular axis. $^{5-7}$ Gelatin contains positively and negatively charged as well as hydrophobic domains folded into a stable, nonnative state that minimizes hydrophobic interactions with water (when dissolved in it). Gelatin can stabilize surfaces by the formation of a steric barrier. ${ }^{8}$ Therefore, the main function of gelatin is as a stabilizer. It is usually used 
in applications such as food processing, ${ }^{9}$ the pharmaceutical industry, ${ }^{7}$ photography, ${ }^{10}$ and electrochemistry. ${ }^{11}$

In many cases, the surface passivation reagents such as polymers and/or surfactant molecules are required to prevent the agglomeration of nanoparticles. ${ }^{12-16}$ For example, Polyvinylpyrrolidone (PVP) has been used as an effective passivation agent for preparing silver nanoparticles (Ag-NPs) and other metal nanoparticles. ${ }^{17}$ One of the green preparation methods of metallic nanoparticles is the polysaccharide method. In this process, Ag-NPs are synthesized in water as an eco-friendly benign solvent with polysaccharides as the stabilizer agent. In some cases, polysaccharides are used as both a reducing agent and a stabilizer. For example, preparation of Ag-NPs using soluble starch was carried out as a stabilizer with $\beta$-D-glucose as a reducing agent in low temperatures. ${ }^{18}$ Ag-NPs are prepared by the reduction of silver cations inside the starch templates. The extensive network of hydrogen bonds in the templates can be passivated by the surface nanoparticles and/or can be protected from aggregation. ${ }^{19}$ This method must normally be done with a gentle heat system because the binding interactions between polysaccharides and metallic nanoparticles are weak and can be reversible with high heat systems, allowing separation from the obtained particles. In this article, we have reported for the first time on the synthesis of Ag-NPs with gelatin as a reducing agent and capping agent. One of the advantages of this method is being able to use renewable materials like gelatin and glucose, which are eco-friendly agents.

\section{Experimental procedure Materials}

Chemicals were analytical grade and used as received without further purification. $\mathrm{AgNO}_{3}$ (99.98\%, Merck KGaA, Darmstadt, Germany), gelatin (type B, Sigma-Aldrich, St Louis, MO, USA), and glucose (BDH Chemical Ltd, Poole, England) were used as silver precursor, capping agent, and reducing agent, respectively. All glassware used in the laboratory experiments was cleaned with a fresh solution of $\mathrm{HNO}_{3} / \mathrm{HCl}(3: 1, \mathrm{v} / \mathrm{v})$, washed thoroughly with doubly distilled water, and dried before use.

\section{Synthesis of gelatin-reduced Ag-NPs}

For synthesis of Ag-NPs, $2.0 \mathrm{~g}$ of gelatin was added to $190 \mathrm{~mL}$ of $\mathrm{H}_{2} \mathrm{O}$ in a flask, and the solution was stirred to obtain a clear solution. Aqueous $\operatorname{AgNO}_{3}(10 \mathrm{~mL}, 1 \mathrm{M})$ was added to gelatin solution with continuous stirring to obtain $\mathrm{Ag}^{+} / \mathrm{gel}-\mathrm{sol}$. The solution obtained was distributed into three cuvettes, and the prepared solutions were stirred and maintained for 48 hours at different temperatures, ie, 28 (AG28), 40 (AG40), and $60^{\circ} \mathrm{C}$ (AG60), respectively.

\section{Synthesis of gelatin-glucose-reduced $\mathrm{Ag}-\mathrm{NPs}$}

For synthesis of Ag-NPs, $2.0 \mathrm{~g}$ of gelatin was added to $190 \mathrm{~mL}$ of $\mathrm{H}_{2} \mathrm{O}$ in a flask, and the solution was stirred to obtain a clear solution. To obtain the $\mathrm{Ag}^{+}$/gelatin solution, the silver solution $(10 \mathrm{~mL}, 1 \mathrm{M})$ was added to the gelatin solution with continuous stirring. Then, $20 \mathrm{~mL}$ of glucose solution $(2 \mathrm{M})$ was added to the $\mathrm{Ag}^{+}$/gelatin solution. The solution obtained was distributed into three cuvettes, and the prepared solutions were stirred and maintained for 48 hours at different temperatures, ie, 28 (AGG28), 40 (AGG40), and $60^{\circ} \mathrm{C}$ (AGG60), respectively. All solutions were kept in dark to avoid any photochemical reactions during the experiment.

\section{Characterization}

The Ag-NPs prepared under the various temperatures were characterized by using ultraviolet-visible (UV-vis) spectroscopy, transmission electron microscopy (TEM), X-ray diffraction (XRD), and atomic force microscopy (AFM). The optical absorption properties of prepared samples were characterized using a Lambda $35^{\circledR}$ (PerkinElmer, Waltham, MS, USA) UV-vis spectrophotometer over the range of 300-700 nm. TEM images were obtained with a Hitachi H-7100 ${ }^{\circledR}$ electron microscope (Hitachi High-Technologies Corporation, Tokyo, Japan), and the particle size distributions of nanoparticles were determined using the UTHSCSA Image Tool ${ }^{\circledR}$ Version 3.00 program (UTHSCSA Dental Diagnostic Science, San Antonio, TX, USA). The XRD patterns were carried out on a Philips X'pert $\left(\mathrm{Cu} \mathrm{K}_{\alpha}\right)$ and were recorded at a scan speed of $2 \% \mathrm{~min}$. The AFM image was obtained on an Ambios Q-scope ${ }^{\circledR}$ (Ambios Technology, Santa Cruz, CA, USA) (SPM) machine.

\section{Results and discussion}

The color of silver solutions in gelatin solutions at various temperature gradually changed from colorless to light brown and then to brown, and in gelatin-glucose solutions changed from colorless to light brown and then to brown and finally dark brown. This indicates the formation of Ag-NPs in both types of solutions. The prepared particles could dissociate due to further laser ablation to form smaller particles stabilized by the amine pendant groups on the gelatin backbone, which leads to the formation of gelatin-stabilized Ag-NPs. Glucose, as the aldehyde, can reduce silver cations to silver atoms and can be oxidized to gluconic acid. The preparation of Ag-NPs was studied by UV-vis spectroscopy, which has 
proven to be a useful spectroscopic method for the detection of prepared metallic nanoparticles. In UV-vis spectra, the Ag-NPs must display a surface plasmon resonance (SPR) band at around $400 \mathrm{~nm}$. The small shift to the left (blue-shift) or to the right (red-shift) in the $\lambda_{\text {max }}$ of the SPR peak could be related to obtaining Ag-NPs at various shapes, sizes, or solvent dependencies of formed Ag-NPs.

After reaction at $28^{\circ} \mathrm{C}$, the Ag-NPs obtained showed a characteristic SPR band for Ag-NPs centered at about
$445 \mathrm{~nm}$ and $438 \mathrm{~nm}$ for AG28 and AGG28, respectively (Figure 1A, D). As shown in Figure 1, the intensity of all the SPR peaks increased as the reaction temperature increased, which indicates the continued reduction of the silver ions, and the increase of the absorbance with the reaction temperature indicates that the concentration of Ag-NPs increased..$^{20,21}$ When the reaction temperatures reached $40^{\circ} \mathrm{C}$ (Figure 1B,E), the absorbance intensities were increased, and the $\lambda_{\text {max }}$ values were slightly blue-shifted to $443 \mathrm{~nm}$ and $431 \mathrm{~nm}$ for

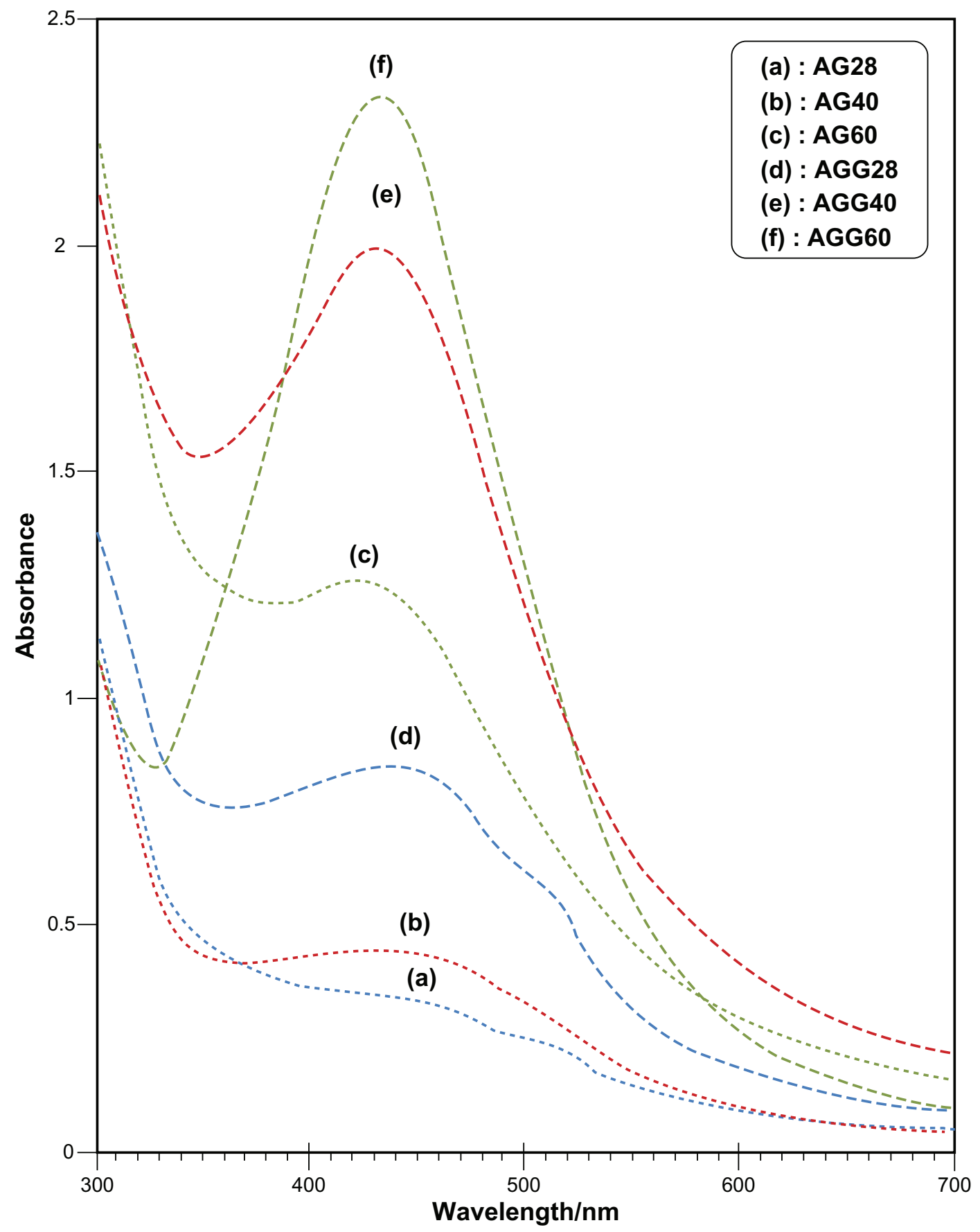

Figure I Ultraviolet-visible absorption spectra of Ag-NPs prepared in gelatin and gelatin-glucose solutions. 

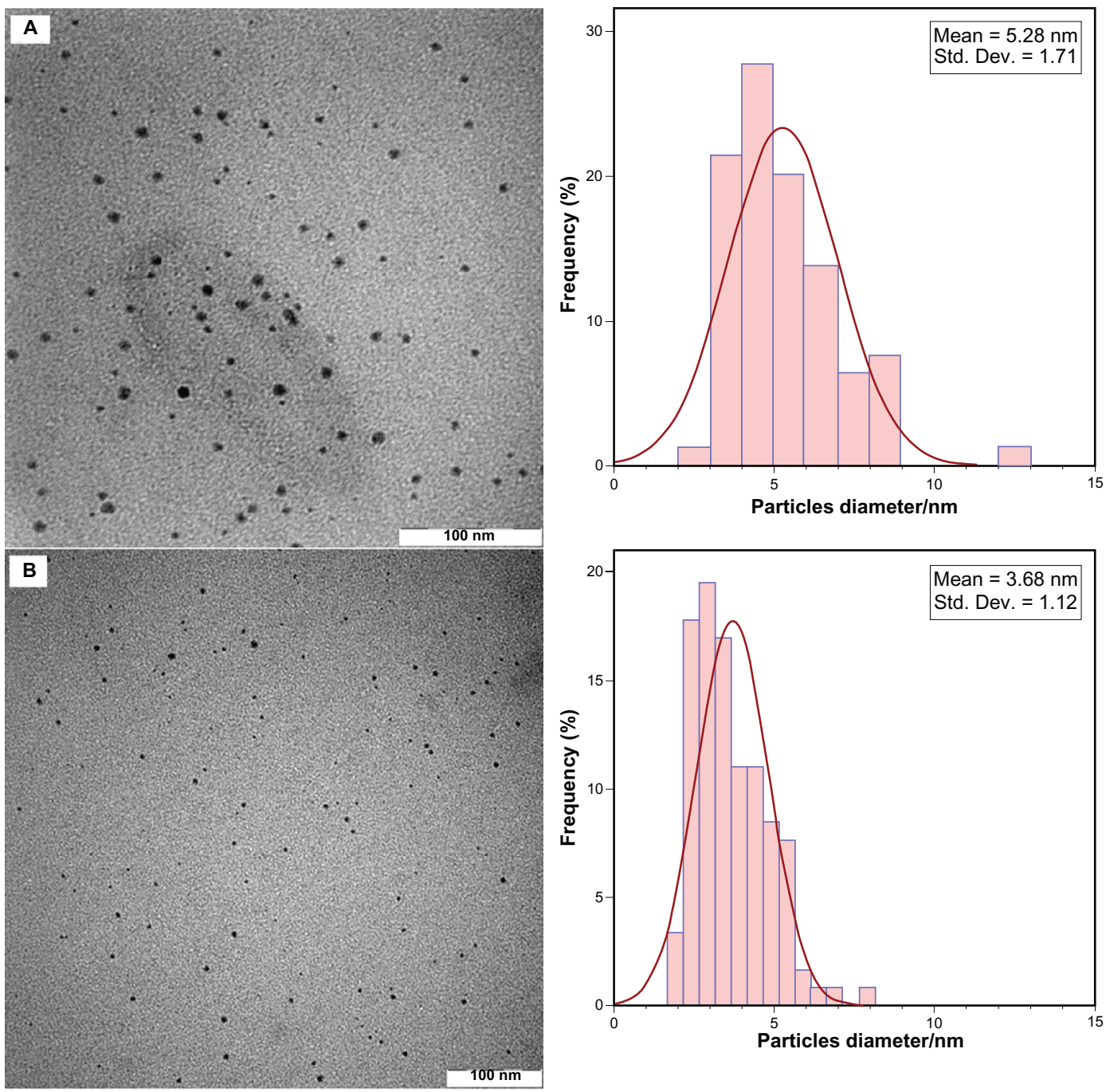

Figure 2 Transmission electron microscopy images and corresponding size distributions of AGG60 (A) and AG60 (B).

AG40 and AGG40, respectively. For AG60 and AGG60 (Figure 1C,F), the absorbance intensities was also increased and blue-shifted to $425 \mathrm{~nm}$ and $430 \mathrm{~nm}$, respectively. This phenomenon indicated that the size of particles was decreased, because the absorbance peak due to the SPR of metallic

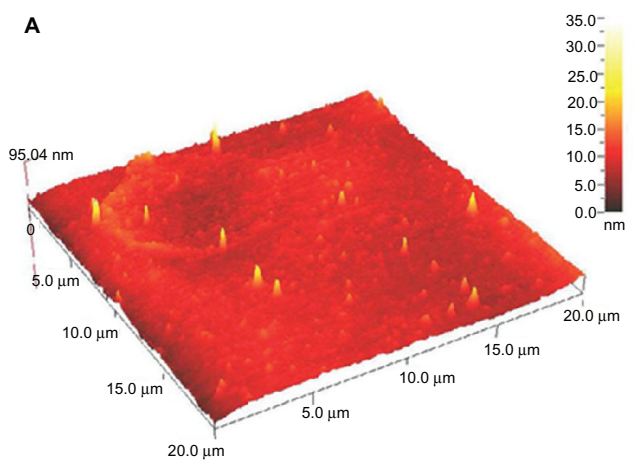

Figure 3 Atomic force microscopy images of AGG60 (A) and AG60 (B). nanoparticles shows the blue-shift with decreasing particle size. ${ }^{22}$ The Ag-NPs could dissociate due to heating to form smaller particles stabilized by the amine pendant groups on the gelatin, which leads to the formation of gelatin-stabilized stable Ag-NPs. ${ }^{23}$ Figure 2 shows typical TEM images and

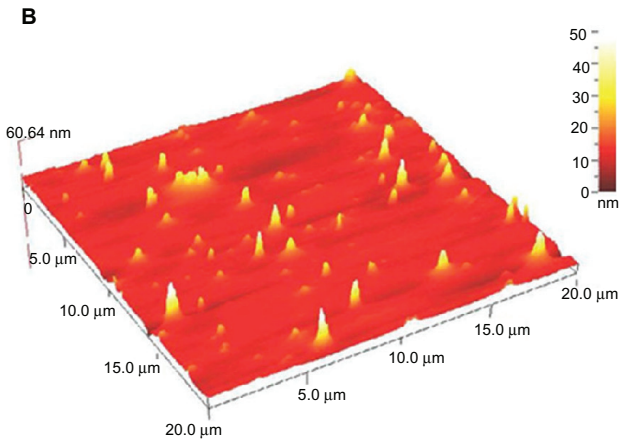




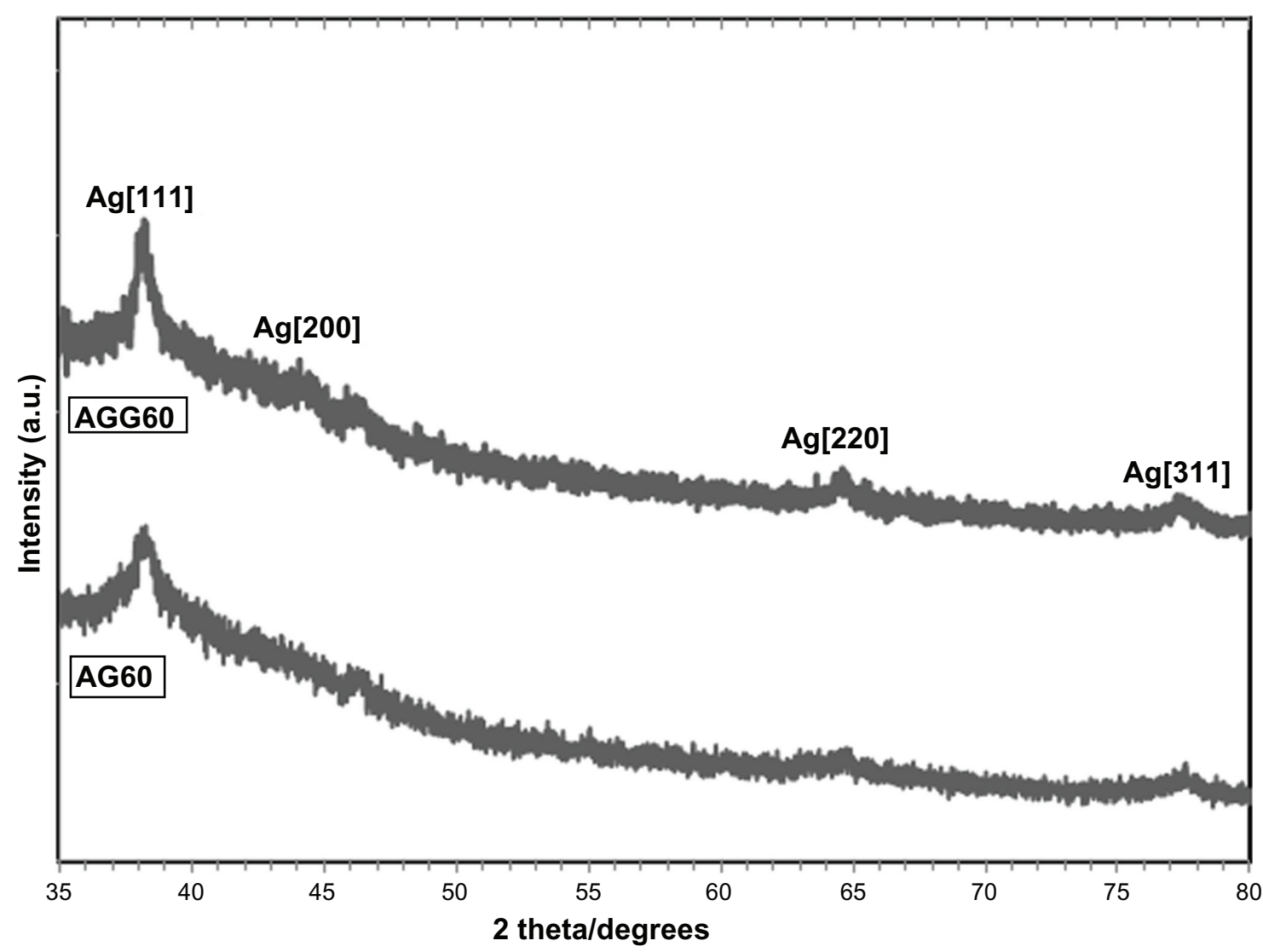

Figure 4 X-ray diffraction patterns of AGG60 and AG60.

the corresponding particle size distribution of the prepared Ag-NPs at different reaction media. The TEM results indicate that the samples obtained in gelatin and gelatin-glucose solutions retained a narrower particle size distribution. It was found that the particle size of Ag-NPs obtained in gelatin solutions is smaller than in the gelatin-glucose solutions, which can be related to rate of reduction reaction. The average size of all prepared Ag-NPs was less than $15 \mathrm{~nm}$, and a smaller average size (about $3.7 \mathrm{~nm}$ ) was obtained for AG60.

The AFM results display the surface morphology of the monodispersed Ag-NPs formed in gelatin and gelatin-glucose media. As observed in Figure 3, the value determined by the AFM was close to that determined by the TEM, and the films of gelatin and gelatin-glucose containing Ag-NPs showed a dense and uniform packed structure. Thus, the Ag-NPs and gelatin and Ag-NPs and gelatin-glucose films could provide a biocompatible and rough surface for biological uses, eg, cell immobilization. Figure 4 shows the XRD patterns of AG60 and AGG60, which show the synthesis of the silver crystalline structure. The XRD peaks at $2 \theta$ degrees of $37.9,44.1,64.3$, and 76.9 and can be attributed to the (111), (200), (220), and (311) crystalline planes of the face centered cubic (fcc) crystalline structure of metallic silver, respectively (JCPDS file No. 00-004-0783).

\section{Conclusion}

Briefly, we have investigated a green method to synthesize Ag-NPs in corresponding metal solution using green agents such as gelatin and glucose without special physical conditions. The Ag-NPs obtained in the presence of gelatin (in the absence of glucose) were smaller in particle size, which can be related to the rate of the reduction process. This preparation method is simple and eco-friendly and may be extended to other noble metals, such as gold ( $\mathrm{Au})$, palladium $(\mathrm{Pd})$, and platinium $(\mathrm{Pt})$, and may possibly find various additional medicinal, industrial, and technological applications.

\section{Disclosure}

The authors report no conflicts of interest in this work.

\section{References}

1. Bhattacharya D, Gupta RK. Nanotechnology and potential of microorganisms. Crit Rev Biotechnol. 2005;25:199-204.

2. Narayanan R, El-Sayed MA. Catalysis with transition metal nanoparticles in colloidal solution: nanoparticle shape dependence and stability. J Phys Chem B. 2005;109:12663-12676. 
3. Eychmuller A. Structure and photophysics of semiconductor nanocrystals. J Phys Chem B. 2000;104:6514-6528.

4. Salata OV. Applications of nanoparticles in biology and medicine. J Nanobiotechnol. 2004;2:3-9.

5. Kawanishi N, Christenson HK, Ninham BW. Measurement of the interaction between adsorbed polyelectrolytes: gelatin on mica surfaces. J Phys Chem. 1990;94:4611-4617.

6. Likos CN, Vaynberg KA, Lowen H, et al. Colloidal stabilization by adsorbed gelatin. Langmuir. 2000;16:4100-4108.

7. Tabata Y, Ikada Y. Protein release from gelatin matrices. Adv Drug Delivery Rev. 1998;31:287-301.

8. Akbulut M, Reddy NK, Bechtloff B, et al. Flow-induced conformational changes in gelatin structure and colloidal stabilization. Langmuir. 2008;24:9636-9641.

9. Ward AG, Courts A. The Science and Technology of Gelatin. London: Academic Press; 1977.

10. Mees KCE. The Theory of Photographic Process. New York: MacMillan; 1966.

11. Brown GM, Hope GA. SERS study of the adsorption of gelatin at a copper electrode in sulfuric acid solution. $J$ Electroanal Chem. 1995;397:293-300.

12. Darroudi M, Ahmad MB, Zamiri R, et al. Preparation and characterization of gelatin mediated silver nanoparticles by laser ablation. J Alloy Compd. 2011;509:1301-1304.

13. Darroudi M, Ahmad MB, Abdullah AH, et al. Effect of accelerator in green synthesis of silver nanoparticles. Int J Mol Sci. 2010;11:3898-3905.

14. Darroudi M, Ahmad MB, Zamiri R, et al. Time-dependent preparation of gelatin-stabilized silver nanoparticles by pulsed Nd: YAG laser. Solid State Sci. 2011 (Article in Press).
15. Zamiri R, Azmi BZ, Darroudi M, et al. Preparation of starch stabilized silver nanoparticles with spatial self-phase modulation properties by laser ablation technique. Appl Phys A. 2011;102:189-194.

16. Shameli K, Ahmad MB, Yunus WMZW, et al. Silver/poly (lactic acid) nanocomposites: preparation, characterization, and antibacterial activity. Int J Nanomed. 2010;5:573-579.

17. Debnath D, Kim C, Kim SH, et al. Solid-state synthesis of silver nanoparticles at room temperature: poly(vinylpyrrolidone) as a tool. Macromol Rapid Commun. 2010;31:549-553.

18. Raveendran P, Fu J, Wallen SL. Completely "green" synthesis and stabilization of metal nanoparticles. J Am Chem Soc. 2003;125:13940-13941.

19. Raveendran P, Fu J, Wallen SL. A simple and "green" method for the synthesis of $\mathrm{Au}, \mathrm{Ag}$, and $\mathrm{Au}-\mathrm{Ag}$ alloy nanoparticles. Green Chem. 2005;8:34-38.

20. Bohren CF, Huffman DR. Absorption and Scattering of Light by Small Particles. New York: John Wiley and Sons Inc.; 1998.

21. Darroudi M, Ahmad MB, Shameli K, et al. Synthesis and characterization of UV-irradiated silver/montmorillonite nanocomposites. Solid State Sci. 2009;11:1621-1624.

22. Heath JR. Size-dependent surface-plasmon resonances of bare silver particles. Phys Rev B. 1989;40:9982-9985.

23. Zhang JJ, Gu MM, Zheng TT, et al. Synthesis of gelatin-stabilized gold nanoparticles and assembly of carboxylic single-walled carbon nanotubes/Au composites for cytosensing and drug uptake. Anal Chem. 2009;81:6641-6648.
International Journal of Nanomedicine

\section{Publish your work in this journal}

The International Journal of Nanomedicine is an international, peerreviewed journal focusing on the application of nanotechnology in diagnostics, therapeutics, and drug delivery systems throughout the biomedical field. This journal is indexed on PubMed Central, MedLine, CAS, SciSearch $\AA$, Current Contents $₫ /$ Clinical Medicine,

\section{Dovepress}

Journal Citation Reports/Science Edition, EMBase, Scopus and the Elsevier Bibliographic databases. The manuscript management system is completely online and includes a very quick and fair peer-review system, which is all easy to use. Visit http://www.dovepress.com/ testimonials.php to read real quotes from published authors. 